\title{
ON THE FLATNESS OF COMPLETE FORMALLY PROJECTIVE MODULES
}

\section{SATOSHI SUZUKI}

In the paper [4], the author introduced the notion of $m$-adic free modules in order to give a differential characterization of regular local rings. Later, more generally, A. Grothendieck introduced the notion of formally projective modules for the similar purpose in his [3]. Comparisons of these two notions in important cases were given in [5].

In the present paper, we intend to study the relationship between the flatness and the formally projectivity. The main purpose is to show the flatness of complete formally projective modules over complete Zariski rings, using results in [5]. As a result of it, we can characterize the formally projectivity of complete modules over complete semilocal rings by the flatness. Meanwhile, we prove the separatedness of finite coefficients extension of complete formally projective modules over complete Zariski rings.

Let $R$ be a commutative ring with an identity and $\mathfrak{m}$ be an ideal. Let $M$ be an $R$-module. We consider the m-adic topologies both in $R$ and $M$. By the completeness of $R$-modules, we always mean the separated completeness.

We follow the terminology in [5]. We begin with a supplement to $[5]$.

The following lemma is a part of Proposition 4.1, $\mathrm{n}^{\circ} 4, \mathrm{IV}$, in [2].

Lemma 1. Assume that $\mathrm{m}$ is nilpotent. Then $M$ is a free module if and only if $M / \mathrm{m} M$ is a free $R / \mathrm{m}$-module and $\operatorname{Tor}_{1}^{R}(M, R / \mathrm{m})=0$.

From this lemma the following corollary follows.

Corollary. Assume that $\mathrm{m}$ is nilpotent and $M$ is a free $R$-module. Let $\left\{a_{i}\right\}_{i \in I}$ be a set of elements in $M$ such that $\left\{\right.$ the class of $a_{i}$ modulo $\mathrm{m} M\}_{i \in I}$ forms a free base of $M / \mathrm{m} M$ over $R / \mathrm{m}$. Then, $\left\{a_{i}\right\}_{i \in I}$ is a free base of $M$.

Proposition. ${ }^{1}$ Assume that $M$ is weakly m-adic free module. Let

Presented to the Society, April 20, 1968 under the title Formally projective modules and ideal adic free modules; received by the editors December 11, 1966.

${ }^{1}$ In the paper [5], we always assumed the existence of a finite base for $\mathrm{m}$. However actually, all assertions in that paper are still valid when we take off this assumption except for Corollary 2. 
$\left\{a_{i}\right\}_{i \in I}$ be a set of elements in $M$ such that $\left\{\right.$ the class of $a_{i}$ modulo $\mathrm{m} M\}_{i \in I}$ forms a free base of $M / \mathrm{m} M$. Then the Hausdorffization $M / \cap_{n=0}^{\infty} \mathfrak{m}^{n} M$ of $M$ is an $\mathrm{nt}$-adic free module and $\left\{\right.$ the class of $a_{i}$ modulo $\left.\mathfrak{m}^{n} M\right\}_{i \in I}$ is an $\mathfrak{m}$-adic free base of $M / \cap_{n=0}^{\infty} \mathfrak{m}^{n} M$.

Proof. By our assumption, $M / \mathfrak{m}^{n} M$ is a free $R / \mathfrak{m}^{n}$-module for each $n=1,2, \cdots$. Hence by the corollary to Lemma 1 , the class of $a_{i}$ modulo $\left.\mathfrak{m}^{n} M\right\}_{i \in I}$ forms a free base of $M / \mathfrak{m}^{n} M$ over $R / \mathfrak{m}^{n}$. This proves our assertion.

Corollary 1. A complete weakly $\mathrm{m}$-adic free module is a complete $\mathrm{m}$-adic free module.

CoRollary 2. A complete formally projective module is a direct summand of a complete $\mathrm{m}$-adic free module.

Proof. This follows from Theorem 1 in [5] and Corollary 1.

The following lemma is a part of Theorem $1, \S 5$, Chapter III in [1].

Lemma 2. If $M$ is flat, $M / \mathrm{m}^{n} M$ is a flat $R / \mathrm{m}^{n}$-module for every $n=1,2, \cdots$. The converse is true in case where $R$ is noetherian and $M$ is ideally separated i.e. $I \otimes_{R} M$ is separated for every ideal I of finite type in $R$ (Definition $1, n^{\circ} 1, \S 5$, Chapter III in [1]).

THEOREM 1. Let $R$ be a noetherian complete $\mathrm{m}$-adic ring and $M$ be a complete formally projective $R$-module. Let $N$ be a finite $R$-module. Then, $N \otimes_{R} M$ is a complete $R$-module (especially, separated $R$-module).

Proof. If we prove that $M^{\prime}=N \otimes_{R} M$ is separated, the completeness of $M^{\prime}$ follows from the finiteness of $N$. By Corollary 2 to Proposition, it is enough to show the separatedness of $M^{\prime}$ in case where $M$ is $\mathrm{m}$-adic free.

Let $\left\{f_{i}\right\}_{i=1,2, \ldots, s}$ be a set of generators of $N$ as an $R$-module. Let $a$ be an element in $\bigcap_{n} \mathfrak{m}^{n} M^{\prime}$. We shall prove that $a=0$. Put $a=\sum_{i=1}^{s} f_{i}$ $\otimes b_{i}$ where $b_{i} \in M(i=1,2, \cdots, s)$. There exists a countable subset $\left\{a_{k} \mid k=1,2, \cdots\right\}$ of $M$ such that:

(1) $\left\{a_{k} \mid k=1,2, \cdots\right\}$ is a part of an $m$-adic free base of $M$, and

(2) for every $i=1,2, \cdots, s, b_{i}$ is expressed as $\sum_{\boldsymbol{k}=1}^{\infty} h(i)_{k} a_{k}$ where the $\left\{h(i)_{k} \mid k=1,2, \cdots\right\}$ are convergent sequences to zero in $R$ in its $\mathrm{m}$-adic topology.

First we shall show that $\sum_{i=1}^{s} h(i)_{k} f_{i}=0$ for all $k=1,2, \cdots$ For each $n=1,2, \cdots$, there exists a positive integer $N_{n}$ such that $h(i)_{k} \in \mathrm{m}^{n}(i=1,2, \cdots, s)$ if $k>N_{n}$. Then

$$
b_{i} \equiv \sum_{k=1}^{N_{n}} h(i)_{k} a_{k} \quad \text { modulo } \mathrm{m}^{n} M \quad(i=1,2, \cdots, s)
$$


Therefore

$$
\begin{aligned}
a=\sum_{i=1}^{s} f_{i} \otimes b_{i} & \equiv \sum_{i=1}^{8} f_{i} \otimes\left(\sum_{k=1}^{N_{n}} h(i)_{k} a_{k}\right) \quad \text { modulo } \mathfrak{m}^{n} M^{\prime} \\
& \equiv \sum_{k=1}^{N_{n}}\left\{\left(\sum_{i=1}^{s} h(i)_{k} f_{i}\right) \otimes a_{k}\right\} \quad \text { modulo } \mathfrak{m}^{n} M^{\prime} .
\end{aligned}
$$

Since $a \in \mathfrak{m}^{n} M^{\prime}$, we have

$$
\sum_{k=1}^{N_{n}}\left\{\left(\sum_{i=1}^{s} h(i)_{k} f_{i}\right) \otimes a_{k}\right\} \equiv 0 \quad \operatorname{modulo} \mathrm{m}^{n} M^{\prime}
$$

On the other hand, $M^{\prime} / \mathfrak{m}^{n} M^{\prime}=\left(N / \mathfrak{m}^{n} N\right) \otimes_{R / \mathfrak{m}^{n}}\left(M / \mathfrak{m}^{n} M\right)$ and \{the class of $a_{k}$ modulo $\left.\mathfrak{m}^{n} M\right\}_{k=1,2, \ldots}$ is a part of a free base of $M / \mathfrak{m}^{n} M$ over $R / \mathfrak{m}^{n}$. Therefore $\sum_{i=1}^{s} h(i)_{k} f_{i} \in \mathfrak{m}^{n} N$ for $k=1,2$, $\cdots, N_{n}$, hence $\sum_{i=1}^{s} h(i)_{k} f_{i} \equiv 0$ modulo $\mathfrak{m}^{n} N$ for all $k=1,2, \cdots$. Hence $\sum_{i=1}^{s} h(i)_{k} f_{i}=0$ for all $k=1,2, \cdots$, as we have asserted.

Thus we have known that the vectors $H_{k}=\left(h(1)_{k}, h(2)_{k}, \cdots, h(s)_{k}\right)$ $(k=1,2, \cdots)$ are elements in the submodule $L$ of the direct sum $T$ of $s$-copies of $R$ such that

$$
\begin{aligned}
L=\left\{(h(1), h(2), \cdots, h(s)) \mid \sum_{i=1}^{s} h(i) f_{i}\right. & =0, \\
& h(i) \in R(i=1,2, \cdots, s)\} .
\end{aligned}
$$

Let $G_{1}, G_{2}, \cdots, G_{t}$ be a set of generators for $L$ with $G_{j}=\left(g(1)_{j}, \cdots\right.$, $\left.g(s)_{j}\right)$ for $j=1,2, \cdots, t$. Then any element $x$ in $\mathfrak{m}^{n} L$ can be written as $x=\sum_{j=1}^{t} l_{j} G_{j}$ with $l_{j} \in \mathrm{m}^{n}$. Since the $H_{k}$ converges to zero in the m-adic topology in $L$, we can write $H_{k}=\sum l_{k}^{j} G_{j}$ with $\left\{l_{k}^{j} \mid k=1,2, \cdots\right\}$ converging to zero in $R$ for each $j$. Therefore, we get our assertion as follows:

$$
\begin{aligned}
a & =\sum_{i=1}^{s} f_{i} \otimes b_{i}=\sum_{i=1}^{s} f_{i} \otimes\left(\sum_{k=1}^{\infty} h(i)_{k} a_{k}\right) \\
& =\sum_{i=1}^{s} f_{i} \otimes\left(\sum_{k=1}^{\infty} \sum_{j=1}^{t} l_{k}^{j} g(i) j^{a_{k}}\right)=\sum_{i=1}^{s} f_{i} \otimes\left\{\sum_{j=1}^{t} g(i)_{j}\left(\sum_{k=1}^{\infty} l_{k}^{j} a_{k}\right)\right\} \\
& =\sum_{j=1}^{t}\left\{\sum_{i=1}^{s} f_{i} \otimes g(i)_{j} \sum_{k=1}^{\infty} l_{k}^{j} a_{k}\right\} \\
& =\sum_{j=1}^{t}\left\{\sum_{i=1}^{s} g(i)_{j} f_{i} \otimes\left(\sum_{k=1}^{\infty} l_{k}^{j} a_{k}\right)\right\}=0 .
\end{aligned}
$$


THEOREM 2. Let $R$ be a complete noetherian $\mathrm{m}$-adic ring and $M$ be a complete formally projective $R$-module. Let $R^{\prime}$ be a finite commutative $R$-algebra. Then $R^{\prime} \otimes_{R} M$ is a complete formally projective $\mathfrak{m} R^{\prime}$-adic module.

PRoof. This follows from Theorem 1.

By Lemma 2 and Theorem 1, we get the following

TheOREM 3. A complete formally projective module over a complete noetherian $\mathfrak{m}$-adic ring is flat.

Theorem 4. Assume that $R$ is a quasi-semilocal ring and $\mathrm{m}$ is a defining ideal of its topology. Then we have the following assertions.

(1) If $M$ is flat, $M$ is formally projective. Conversely,

(2) Assume that $R$ is noetherian and both $R$ and $M$ are complete. Then, if $M$ is formally projective, $M$ is flat.

Proof. (2) is included in Theorem 3. We shall prove (1). We have only to prove that $M / \mathfrak{m}^{n} M$ is a projective $R / \mathfrak{m}^{n}$-module for each $n=1,2, \cdots$. It is enough to show that in case $n=1$. Let $\mathfrak{m}_{i}$ $(i=1,2, \cdots, l)$ be maximal ideals in $R$. Then there exists a positive integer $h$ such that $\mathfrak{m} \supset \mathfrak{m}_{1}^{h} \mathfrak{m}_{2}^{h} \cdots \mathfrak{m}_{l}^{h}=\mathfrak{a}$. We have only to prove the projectivity of $M / \mathfrak{a} M$ as an $R / \mathfrak{a}$-module. By Lemma $2, M / \mathrm{m}_{i}^{h} M$ is a flat $R / \mathfrak{m}_{i}^{h}$-module. Hence $\operatorname{Tor}_{1}^{R / \mathfrak{m}_{i}^{h}}\left(M / \mathfrak{m}_{1}^{h} M, R / \mathfrak{m}_{i}\right)=0$. Therefore by Lemma $1, M / \mathfrak{m}_{i}^{h} M$ is a projective $R / \mathfrak{a}$-module, because $R / \mathrm{m}_{i}^{h}$ is a direct summand of $R / \mathfrak{a}$. Therefore $M / \mathfrak{a} M=\bigcap_{i=1}^{h} M / \mathrm{m}_{i}^{h} M$ is a projective $R / \mathfrak{a}$-module, which proves our theorem.

\section{REFERENCES}

1. N. Bourbaki, Eléments de mathematique. XXVIII: Algébre commutative, Chapters 3 and 4, Hermann, Paris, 1961.

2. A. Grothendieck, Seminaire de géométrie algébrique, Inst. Hautes Etudes Sci. Publ. Math. 1960-1961.

3. - Éléments de géométrie algébrique. IV, Inst. Hautes Études Sci. Publ. Math. 20 (1964).

4. S. Suzuki, Some results on Hausdorff m-adic modules and m-adic differentials, J. Math. Kyoto Univ. 2 (1963), 157-182.

5. - Note on formally projective modules, J. Math. Kyoto Univ. 5 (1966), 193-196.

Queen's University, Kingston, Canada 